\title{
NEOGEOGRAFÍA, BIG DATA Y TIG: PROBLEMAS Y NUEVAS POSIBILIDADES
}

\author{
Joaquín BOSQUE SENDRA (joaquin.bosque@,uah.es)
}

Recibido: $17 / 03 / 2015$

Aceptado: 15/07/2015

$\boldsymbol{R} \boldsymbol{E S U M E N :}$ La Neogeografía y el BIG data están cambiando la manera de investigar en Geografía, el desarrollo de estos enfoques se basa mucho en la aparición de novedades tecnológicas, en particular de nuevas tecnologías de la información geográfica: GPS, SIG, etc.

PALABRAS CLAVE: Neogeografia, GPS, SIG, Ciencia ciudadana, BIG data.

\section{NEOGEOGRAPHY, BIG DATA AND TIG: PROBLEMS AND POSSIBILITIES}

ABSTRACT: The Neogeography and the BIG data are changing the way research in geography, the development of this approach is very much based on the emergence of new technologies, in particular in new technologies of geographic information: GPS, GIS, etc.

KEY WORDS: GPS, GIS, Neogeography, science citizen, BIG data.

En los últimos años han surgido nuevas maneras de realizar estudios y aportaciones geográficas, las denominadas Neogeografía, Geografía voluntaria, y quizá otras similares, que han ampliado bastante el tradicional enfoque de la disciplina de la Geografía. Este trabajo intenta plantear y analizar brevemente estos temas y, en especial, sus conexiones con las Tecnologías de la información geográfica.

\section{DEFINICIONES DE NEOGEOGRAFÍA}

La Neogeografía, y/o la Geografía voluntaria, se puede considerar que constituyen un nuevo saber geográfico a añadir a los otros tres o cuatro que ya planteó Y. Lacoste hace años (la geografía de los profesores, la geografía de los estados mayores,...). Según M. GoODCHILD (2007) la Neogeografia/Geografía voluntaria se define como la participación de actores voluntarios, a veces con ninguna formación previa en cuestiones geográficas, en la elaboración de datos territoriales que se ponen al servicio de todos en Internet. Sin embargo otras personas definen la Neogeografía de un modo más amplio, y, 
desde ciertos puntos de vista, más discutible: para TURNER (2006) la Neogeografía consiste en personas realizando sus propios mapas, usando para ello herramientas sencillas y asequibles, disponibles libremente en Internet, y generando, con todo ello, un conocimiento del territorio similar al obtenido por los geógrafos profesionales y académicos, empleando, en este caso, SIG y otras herramientas más costosas y complicadas de manejar. En otras palabras, según esta opinión, la Neogeografía sustituye a los SIG (TURNER, 2009) y a los propios profesionales que los utilizan, y construye una información geográfica de calidad similar a la obtenida por ellos.

Aunque existen elementos en común en ambas definiciones también, es evidente, que se observan importantes diferencias (WILSON y GRAHAM, 2013); el criterio de GOODCHILD (2009) parece, a los geógrafos académicos (como el autor de este texto), más razonable. Para la Geografía académica, la Geografía voluntaria produce datos territoriales, no tanto información geográfica, que necesita para ello una actividad más compleja y basada en teorías e ideas elaboradas, en ELWOOD y OTROS (2012) se puede encontrar un análisis detallado de estas ideas. No obstante, no debemos dejar de considerar la posible verdad que subyace a que la Neogeografía esté no solo generando datos geográficos en masa, sino que, junto a ellos, además aporte alguna nueva información, nuevos conocimientos territoriales producto de la elaboración de esos datos.

La aparición de este nuevo saber geográfico no es tan extraña; en muchas otras especialidades científicas han surgido iniciativas semejantes. Se trata, en realidad, de un ejemplo de lo que se suele denominar Ciencia ciudadana, que en algunos casos, como, por ejemplo, en la Ornitología tiene una muy larga tradición. Las disciplinas basadas en la observación, como la ya mencionada Ornitología o la Astronomía, donde los astrónomos aficionados aportan bastante datos a la práctica de los profesionales (ver, por ejemplo, elobservadordeestrellasdobles.wordpress.com/), son especialmente susceptibles de que se realicen aportaciones de no profesionales. En alguna medida, la Geografía estaría dentro de este tipo de disciplinas.

De hecho ya se han institucionalizado estas iniciativas de ciencia participativa, por ejemplo ver el portal www.socientize.eu/ o www.csic.es/libro-verde-de-la-ciencia-ciudadana. En CONNORS y OTROS (2012) se plantea en detalle esta relación Neogeografía y Ciencia ciudadana. En castellano, Capel (2010) también ha tratado estas cuestiones.

Una iniciativa territorial muy relevante en este sentido es el Open Street Map (www.openstreetmap.es/) que está elaborando un amplio mapa callejero de todas, o de muchas, ciudades del mundo, la universidad española también 
participa en esta acción: https://diariodigital.ujaen.es/node/45767. Además se pueden citar muchas otras iniciativas de lo que suele denominar «cartografía colaborativa», como muestra se pueden mencionar dos blog en los que plantean estos temas, el de Madri+D: http://www.madrimasd.org/blogs/mapasproyectosculturales/tag/cartografiacolaborativa/ o el de blogpolis: http://cordopolis.es/descoordenado/tag/cartografia-colaborativa/. Una de las más importantes aplicaciones de este tipo de cartografía puede ser en la Cooperación al desarrollo, una bonita muestra se puede ver en la comunicación de PRIETO CERDÁN Y OTROS (2014).

\section{PAPEL DE LAS TIG Y DE LOS SIG EN LA NEOGEOGRAFÍA.}

¿Cuáles son las razones, las causas, de la aparición de estos nuevos saberes científicos, y, en particular, del surgimiento de la Neogeografía? Se puede pensar que la influencia principal ha sido el desarrollo de diversas tecnologías.

En primer lugar, de las tecnologías de la comunicación que han facilitado el desarrollo de la WEB como instrumento de contacto y de relación entre millones de personas. Sin ello, por ejemplo, el interés de generar datos geográficos resultaría menor al no poder difundirlos de inmediato y al no llegar a los miles o millones de usuarios en todas partes del mundo.

Pero, para el caso de la Neogeografía, el factor decisivo ha sido la aparición de nuevas tecnologías de la información geográfica que facilitan en gran medida la obtención de datos territoriales. Entre estas se puede mencionar, en primer lugar, el GPS, Global Positioning System, que facilita a todo el mundo conocer las coordenadas del lugar donde se encuentra, tarea que hasta hace unos pocos decenios requería disponer de herramientas y, sobre todo, de conocimientos técnicos elaborados; de hecho no se debe olvidar que el problema de la longitud geográfica ha sido una cuestión científica clave y que ha costado enormes esfuerzos de resolver (SANTOS, 2003). La disponibilidad masiva del GPS en todo tipo de aparatos, incluidos teléfonos móviles, facilita que cualquiera pueda obtener datos precisos de la localización de cualquier cosa, lo que conduce a la fácil y barata creación de datos geográficos por una persona no conocedora de mucha Geografía.

Aparte del GPS, otras tecnologías de la información geográfica, como la Teledetección, la Fotografía aérea, cada vez más asequibles y cuyos resultados suelen estar disponibles en Internet, facilitan que un usuario inexperto pueda retocar, manipular esas imágenes para construir sus propios mapas, sus propios datos geográficos, añadiéndoles, si es necesario, datos GPS u otros. En este sentido la aparición de Google Earth 
(http://www.google.es/intl/es/earth/index.html) y de otros desarrollos similares, ha supuesto una novedad esencial que ha impulsado aún más este proceso.

En resumen, las innovaciones técnicas han facilitado los medios para que sea asequible, barato y fácil, crear, por cada persona interesada, sus propios mapas, sus propios datos geográficos y que, además, los pueda publicar sin costes para que otros los vean y los utilicen.

\section{EL BIG DATA Y LA NEOGEOGRAFÍA}

Por otra parte, se puede considerar que la Neogeografía es solo un ejemplo de lo que ahora se denomina BIG data (PUMAIN, 2014; GRAHAM y SHELTON, 2013; KitChIN, 2013), es decir la explosión de nuevos datos personales, y de otros muchos tipos, que la actividad diaria de personas y de cosas genera de continuo. La razón principal es que, de muchas formas distintas, las personas y las cosas, sobre todo en las ciudades, están monitorizadas continuamente, generando datos, muchos, o casi todos ellos, georreferenciados y que se almacenan en diversos repositorios, unos públicos y otros más privados.

Un ejemplo evidente de esto es la información que el usuario de una tarjeta de crédito, ahora en el bolsillo de cualquiera, genera al ser empleada: la mercancía adquirida, el establecimiento y la cadena comercial a la que pertenece, la posición geográfica de dicho establecimiento, la hora de uso, etc. Todos esos datos son de gran interés para un estudio de Marketing y es de suponer que así sean empleados de continuo por las compañías emisoras de estas tarjetas.

De manera similar otra multitud de actividades diarias de personas, automóviles, y aparatos de todo tipo, generan datos diversos que muestran qué hacen y dónde lo hacen. A todo ello se unen la gran cantidad de sensores instalados en las ciudades modernas: cámaras de vigilancia, controladores de abonos en los transportes públicos, conteos de pasos de automóviles, etc. Por otra parte, las redes sociales existentes en Internet (Facebook, Twitter, etc.) también recogen, por la participación en ellos de los usuarios, numerosos datos personales que permiten estudiar procesos sociales de diverso tipo, muchos de ellos con referencias espaciales.

En resumen, un enorme volumen de información, en gran medida territorial o con una referencia espacial, está disponible en la actualidad y ya se empieza a utilizar de diversas formas; las iniciativas de Smart Cities serían un ejemplo de uso de muchos de estos datos para intentar gestionar de modo más eficiente la vida de una ciudad, se trata de un tema muy extenso y complejo pero de gran interés y porvenir en los próximos años y que, es seguro, que debe incidir, 
de alguna manera, en la investigación realizada por la Geografía académica. Como una muestra de la importancia del tema ver, por ejemplo, algunos congresos dedicados a la cuestión: la conferencia sobre Ciencia de ciudades: citysciences-conference.com/ o el congreso mundial sobre la cuestión que se ha celebrado en 2015 en Barcelona: http:/www.smartcityexpo.com/congress1).

\section{LOS DATOS DEL BIG DATA Y SU EMPLEO}

La disponibilidad de estos numerosos datos genera, o lo debería hacer, una serie de modificaciones en la actividad de los geógrafos académicos que estudian el territorio, en particular aquellos que emplean para esta tarea las tecnologías de la información geográfica.

Hasta este momento, el uso de los SIG (la técnica geográfica especializada en el análisis territorial) tenía su más importante dificultad en la generación de la base de datos a emplear para ello. La obtención de datos era el principal «cuello de botella» que dificultaba un análisis territorial potente y eficaz para resolver problemas. Los SIG tenían incorporadas funciones muy útiles para el manejo de datos y tampoco era muy complicado integrar o relacionar los SIG con modelos predictivos, o de simulación o de optimización (AGUILERA y OTROS, 2010). El problema eran los datos sobre los que aplicar esos procedimientos, su obtención y mantenimiento era costoso y laborioso.

Ahora los datos empiezan a abundar y son mucho más fáciles de conseguir e integrar en un SIG. No solo los datos más tradicionalmente empleados en los SIG están fácilmente disponibles; las iniciativas de creación de las denominadas Infraestructura de datos lo facilitan cada vez más (www.idee.es/), además, en buena medida, los nuevos aspectos del Big data, de la Neogeografía, aportan datos, muchos y muy variados, y, en gran medida, diferentes, a los anteriormente usados en un SIG. Esto cambia la situación. Aunque es evidente que todavía no se han desarrollado los procedimientos y, más aún, la actitud en los geógrafos académicos para intentar utilizar estos nuevos datos, y por ello todavía su uso no está generalizado, pero ya se pueden mencionar algunos ejemplos y experiencias: SHELTON y OTROS (2014) sobre el uso de Twitter; GRAHAM y ZOOK (2013), sobre la Geoweb.

Los nuevos datos parecen más apropiados para mostrar algunas de las decisiones individuales (referentes, por ejemplo, a compras, movimientos y acceso a lugares, etc.) con su componente espacial. Los datos más tradicionales eran adecuados para ver cuál es la situación territorial en un momento del tiempo, el aspecto dinámico era más difícil de medir con ellos. Justo lo contrario de lo que se puede pensar de muchos de los nuevos datos ahora disponibles. 
Estos datos parecen, por lo tanto, mucho más convenientes para conocer el aspecto espacial de las decisiones de las personas, y de los distintos agentes sociales, en el territorio.

Los nuevos datos muestran procesos y no estructuras «estables», es decir lo contrario de los datos usuales de la Geografía, los hasta ahora estudiados con SIG. ¿Es una oportunidad para pasar a estudiar de manera más adecuada los procesos espaciales hasta ahora muy poco tratados en los estudios con SIG?

La respuesta a esta interesante cuestión es difícil. Se puede considerar que existe una falta de métodos de análisis apropiados para el análisis de los procesos socio - espaciales usando un SIG, que están bien preparados, por otra parte, para estudiar las estructuras espaciales pero no tanto para los procesos que las generan.

Por ello, los SIG (y los modelos de análisis actuales) resultarían útiles como herramientas auxiliares para visualizar los nuevos datos, pero no tanto para analizarlos.

Se plantea, por lo tanto, la urgente necesidad de elaborar nuevos modelos de análisis que permitan el estudio de los procesos sociales que ocurren en el territorio y que son los que dan origen a las estructuras espaciales, las que hasta ahora han tratado y estudiado los SIG.

No podemos saber con exactitud cuáles serán esos nuevos métodos, pero posiblemente los modelos basados en Multiagentes (CANTERGIANI, 2011) sean una posible solución, al menos inicial, a este problema metodológico. No obstante, no se deben menospreciar las dificultades ya existentes, y bastante bien conocidas, para integrar SIG y multiagentes. Se trata, por lo tanto, de una tarea pendiente de gran interés y utilidad práctica.

En cualquier caso el impacto del Big data sobre la Geografía es una cuestión todavía imprecisa y, peor aún, difícil de precisar, habrá que esperar tiempo y dedicar mucha atención al tema para poder ver todas las implicaciones que este nueva situación tiene en la Geografía académica y en la investigación geográfica.

\section{LA WIKIFICACIÓN DEL SIG}

La intervención masiva de personas, de aficionados, de no-profesionales, en la creación de datos geográficos, usando para ello las capacidades que permiten las nuevas tecnologías de la comunicación e Internet, no es la única modificación de este tipo que afecta a las tecnologías de la información geográfica y, muy en particular, a los SIG. Algo similar ocurre con los otros tres componentes de un SIG: el hardware, el software y el personal, lo que ha conducido 
a que se empiece a hablar de la denominada «wikificación» del SIG (SUI, 2008).

Respecto al hardware, un buen ejemplo es la aparición de los programas de cálculo en colaboración, la denominada computación distribuida, a través de Internet, de los cuales el ejemplo más conocido sería el salvapantallas SETI@home (setiathome.berkeley.edu/), que ha permitido que millones de personas pongan sus ordenadores personales al servicio del análisis de datos para intentar identificar señales de inteligencia extraterrestres; lo que posteriormente facilitó la creación del proyecto de computación distribuida BOINC que ha propiciado que decenas de importantes cuestiones científicas puedan ser procesadas en ordenadores personales de todo el mundo para ayudar a resolver las más variadas temáticas (ver, boinc.berkeley.edu/). En este sentido, otro avance técnico importante es el desarrollo de la computación en grid (www.gridcomputing.com/) que en Geografía, o más bien en la denominada Geocomputación (BOSQUE SENDRA, 2005), puede ser de gran ayuda para resolver problemas geográficos de difícil tratamiento en la actualidad (GUAN y otros, 2006). Una posible aplicación de estos procedimientos podría ser la resolución de modelos de localización-asignación para tratar cuestiones de localización óptima que ahora presentan problemas para ser solucionados con los programas informáticos y los ordenadores disponibles (MORENO y BOSQUE, 2010).

El software sería otro componente de un SIG que está viendo aumentar de manera sustancial la colaboración de voluntarios, de no-profesionales, que participan en la elaboración del denominado software SIG libre (www.freegis.org/), abierto, que realiza las mismas o muy parecidas funciones que un programa SIG comercial (ver STEINIGER y BOCHER, 2009, para un balance de la situación reciente de este tipo de programas). Los programas de este carácter son muy diversos, existe, en particular, una iniciativa española que ha tenido gran éxito, el desarrollo de gvSIG (www.gvsig.com/es).

En menor medida, respecto al cuarto componente de un SIG, el personal, también se podría hablar de la participación de voluntarios que usan SIG para realizar tareas de ayuda a la población en diversas circunstancias (www.giscorps.org/).

En resumen, la participación masiva de personas voluntarias en la realización de muchas y variadas tareas relacionadas con el territorio y la Geografía, está cambiando de manera sustancial la investigación geográfica. La Geografía académica, en particular la española, no ha reaccionado aún de manera suficiente a estas modificaciones, que claramente son una oportunidad y un 
desafío. Una oportunidad debido a que, seguramente, pueden facilitar un mucho mejor conocimiento del territorio y su dinámica. Un desafío ya que, para poder afrontar esa oportunidad, será necesario realizar cambios profundos en la docencia y en la manera de enfocar la investigación por parte de los geógrafos académicos, y realizar cambios profundos nunca es sencillo ni cómodo.

\section{BIBLIOGRAFÍA}

Aguilera, F.; Gómez Delgado, M. y CANTERGiani, C.C. (2010): «Instrumentos de simulación prospectiva del crecimiento urbano», Ciudad y Territorio. Estudios Territoriales, Vol. XLII ( $\left.{ }^{\circ} 165-166\right), 481-495$.

BOSQUE SENDRA, J. (2005): «Espacio geográfico y ciencias sociales. Nuevas propuestas para el estudio del territorio», Investigaciones regionales, 2005, $\mathrm{n}^{\circ}$ 6, 203-224. http://www.aecr.org/images/ImatgesArticles/2007/10\%20Bosque.pdf

CAPEL, H. (2010): «Geografía en red a comienzos del Tercer Milenio. Por una ciencia solidaria y en colaboración». Scripta Nova. Revista Electrónica de Geografía y Ciencias Sociales. [En línea]. Barcelona: Universidad de Barcelona, 1 de febrero de 2010, vol. XIV, no 313 [ISSN: 1138-9788 1138-9788]

http://www.ub.es/geocrit/sn/sn-313.htm

CANTERGIANI, C.C. (2011): «Modelos basados en agentes aplicados a estudios urbanos: una aproximación teórica», Serie Geográfica, 17 (2011), 29 - 43.

http://dspace.uah.es/dspace/bitstream/handle/10017/14343/29_43_modelos_Carvalho SG 2011 N17.pdf? sequence $=1$ \&isAllowed $=\mathrm{y}$

ConNors, JP, S. L. y MAGGI, K. (2012): «Citizen Science in the Age of Neogeography: Utilizing Volunteered Geographic Information for Environmental Monitoring», Annals of the Association of American Geographers, 102(6) 2012, $1267-$ 1289.

Elwood, S.; GoodchILD, M.F. y SuI, D.Z. (2012): «Researching Volunteered Geographic Information: Spatial Data, Geographic Research, and New Social Practice» Annals of the Association of American Geographers, 102(3) 2012, 571-590.

GoODCHILD, M.F. (2007): «Citizens as sensors: the world of volunteered geography» GeoJournal, Volume 69, Issue 4, 211-221.

GoODCHILD, M.F. (2009): «NeoGeography and the nature of geographic expertise», Journal of Location Based Services, 3: 2, 82-96. DOI: $10.1080 / 17489720902950374$. dx.doi.org/10.1080/17489720902950374

GrAHAM, M. y T. SHELTON (2013): «Geography and the future of big data, big data and the future of geography» Dialogues in Human Geography, November 2013 vol. 3 no. $3,255-261$.

http://dhg.sagepub.com/content $/ 3 / 3 / 255$.abstract

GRAHAM, M. y ZOOK, M. (2013): «Augmented realities and uneven geographies: exploring the geolinguistic contours of the web» Environment and Planning A 45(1) 77- 99.

Guan, Q.; Zhang, T. y Clarke, K.C. (2006): «GeoComputation in the Grid Computing Age» en J. D. CARSwell y T. TeZuKa (Eds.): W2GIS 2006, LNCS 4295, 237-246, 2006. 
link.springer.com/chapter/10.1007/11935148_22\#page-1 http://www.dl.kuis.kyoto-u.ac.jp/w2gis2006/session6/69-guan w2gis.pdf

KitchIN, R. (2013): «Big data and human geography: Opportunities, challenges and risks» Dialogues in Human Geography, 3(3), 262-267. http://eprints.maynoothuniversity.ie/5366/1/RK big\%20data\%20human.pdf

MoRENO JiMÉNEZ, A. y BosQue SENDRA, J. (2010): «Los modelos de localización óptima como herramientas para la planificación territorial y urbana de instalaciones y equipamientos» Ciudad y territorio. Estudios territoriales, número 165-166, 461-480.

Prieto Cerdán, A.; Castillo Salcines, V.; Mira Martínez, J.M.; Mas Martil, R.; BAÑo SÁNCHEZ, J.L. (2014): «Cooperación internacional al desarrollo: cartografía colaborativa en los sectores de Rukara y Huye (Rwanda)». XVI Congreso Nacional de Tecnologías de la Información Geográfica 25, 26 y 27 de Junio de 2014. Alicante.

http://rua.ua.es/dspace/bitstream/10045/46758/1/2014_PrietoCerdan_etal_Congr eso TIG.pdf

PumaIn, D. (2014): «Observation, observation, observation», Cybergeo: European Journal of Geography [En ligne], Editoriaux, mis en ligne le 07 mars 2014, consulté le 12 novembre 2014.

cybergeo.revues.org/26248

SANTOS PÉREZ, J.L. (2003): «El problema de la longitud geográfica» Catastro, $\mathrm{n}^{\circ} 49$, 2003, 133-147.

www.catastro.meh.es/documentos/publicaciones/ct/ct49/luisjsantos.pdf

Shelton, T.; PoOrthuis, A.; Graham, M.; ZooK, M. (2014): «Mapping the data shadows of Hurricane Sandy: Uncovering the sociospatial dimensions of 'big data'», Geoforum, 52, 167-179.

http://dx.doi.org/10.1016/j.

STEINIGER S. y BOCHER E., (2009): «An overview on current free and open source desktop GIS developments». International Journal of Geographical Information Science, 23(10):1345-1370

SUI, D.Z. (2008): «The wikification of GIS and its consequences: Or Angelina Jolie's new tattoo and the future of GIS» Computers, Environment and Urban Systems 32 (2008) $1-5$. http://www.sciencedirect.com/science/article/pii/S0198971507000919

TURNER, AJ (2006): Introduction to Neogeography O'Reilly Media, Inc. ISBN: 9780-596-52995-6 Release Date: December 15, 2006. http://shop.oreilly.com/product/9780596529956.do

TURNER, AJ (2009): How-neogeography-killed-gis. es.slideshare.net/ajturner/how-neogeography-killed-gis

WILSON, M. y Mark GRAHAM (2013): «Neogeography and volunteered geographic information: a conversation with Michael Goodchild and Andrew Turner» Environment and Planning $A$ 2013, volume 45, pages 10-18. doi:10.1068/a44483 